\title{
Extracellular RNA in systemic lupus erythematosus
}

\author{
Shanshan Liư ${ }^{1 \dagger}$, Dandan Wang ${ }^{1 \dagger}$ and Yuan Liư ${ }^{2 *}$
}

\begin{abstract}
Since the discovery of extracellular RNA (exRNA), it has been shown that exRNAs play a significant role as a transducer in intercellular communication and biomarkers in various diseases. Systemic lupus erythematosus (SLE) is a kind of autoimmune disease that has protean manifestations. The survival and long-term prognosis of patients with SLE has improved in these 5-10 years, while disease pathogenesis is still not clear. Many researchers found the changes in exRNA profile, and exRNAs are likely participating in the process of SLE. In this review, we summarize the current profile and function of exRNA in SLE. Circulating miRNAs, in particular, have been identified as biomarkers for SLE diagnosis. We also explore the function of InCRNA in SLE and the potential correlation with disease progression and activity. These studies show that exRNAs may take parts in the process of SLE and some of them can be used as diagnostic tool for SLE.
\end{abstract}

Keywords: exRNAs, miRNAs, IncRNAs, Systemic lupus erythematosus

\section{Background}

The role of extracellular RNA in the pathogenesis and therapy in systemic lupus erythematosus has been extensively addressed. This review will summarize the most recent contents in this field.

\section{Main text \\ Introduction of extracellular RNAs}

Extracellular RNAs (exRNAs) are known as RNA species that are present outside of the cell, in the circulation, or tissue culture supernatants. With the development of sequencing technology, the profiles of exRNAs have been found almost in all bodily fluid and secretions. Moreover, exRNAs that have been found in extracellular fluid contain almost all types of RNA, mRNA transcripts and non-coding RNAs (ncRNA), which include small nuclear RNAs (snRNA), microRNAs (miRNA), Y-RNAs, long non-coding RNAs (lncRNA), small nucleolar RNAs (snoRNA), ribosomal RNAs (rRNA), circular RNAs (circRNA), piwi-RNAs (piRNA), transfer RNAs (tRNA), 5' -tRNA halves, and microbial

\footnotetext{
* Correspondence: yliu@gsu.edu

'Shanshan Liu and Dandan Wang contributed equally to this work.

${ }^{2}$ Program of Immunology \& Molecular Cellular Biology, Department of Biology, Center for Diagnostics \& Therapeutics, Center of Inflammation, Immunity \& Infection, Georgia State University, Atlanta, GA 30302, USA Full list of author information is available at the end of the article
}

RNAs [1-13]. Among the various types of RNA, many exRNAs sequencing are focused on small RNA species $(<200 \mathrm{nt})$, especially miRNA $[14,15]$. The range of concentration of miRNA in sequencing of exRNA varied from 40 to $75 \%$ in human serum and plasma $[1,6,13]$. MiRNAs were identified an average of $21.7 \%$ ranging in size from 15 to $40 \mathrm{nt}$ in human seminal fluid [10]. However, miRNA transcript present different forms in kinds of bodily fluids. For example, many results in published reports show that the miRNA expression profile of cerebrospinal fluid (CSF) is substantially different from that of serum [16]. These results indicate that some extracellular miRNA may be tissue-dependent.

More recently, it has been reported that exRNAs are packaged into complexes with some carriers such as extracellular vesicles (EVs), lipoproteins (LPPs) including LDLs [17, 18], HDLs [18], and ribonucleoprotein particles (RNPs), such as Ago2-containing RNPs [19, 20]. The diameter of EVs ranges in size from $\sim 30 \mathrm{~nm}$ to 400 $\mathrm{nm}$ according to the type [21-24]. The biogenesis of EVs includes a range of complex mechanisms, which is still not clear enough. Based on their biogenesis pathways, EVs includes exosomes, microvesicles and apoptotic bodies, each of those being highly heterogeneous in their own [25-27]. The molecular composition of EVs contains mRNAs, ncRNAs, proteins, and lipids $[25,26]$. 
Cells release EVs to communicate with other neighboring cells. EVs can modify the physiological state of target cell via receptor-ligand interaction, endocytosis or phagocytosis [28]. The secretion of EVs is a conservative process throughout evolution [29], and it is reported that EVs can be released by cells from all eukaryotes and some prokaryotic cells. EVs have both physiological and pathological roles in cell-to-cell signaling, immune response, and age-related disease, including neurodegenerative, cardiovascular diseases and cancer [30-36].

\section{Extracellular RNA function}

ExRNAs contain many types of ncRNA, such as miRNAs and LncRNAs. MiRNAs are a class of small non-coding RNA which negatively regulate target genes through binding gene sequences, about 21-25 nucleotides in length. Recently, a significant number of miRNA have been found in human bodily fluids by using next-generation sequencing (NGS). The abnormal expression of miRNAs affects cell function and processes, such as apoptosis or proliferation, and links between miRNAs and cancer development exist [37]. Accordingly, some circulating miRNAs in plasma, serum, urine, or saliva have been changed specifically and successfully taken as biomarkers for several diseases. For example, 15 serum miRNAs were up-regulated in prostate cancer comparing to healthy individuals, including miR-16, miR-92a, miR-103, miR-107, miR-197, miR-34b, miR-328, miR-485-3p, miR-486-5p, miR-92b, miR-574-3p, miR-636, miR-640, miR-766, and miR-885-5p [38]. And, exosomal miR-141 is up-expressed in the serum from patients of prostate cancer compared with the healthy volunteers, and detected as a useful biomarker for the diagnosis of metastatic prostate cancer [39]. The levels of miR-126 and miR-182 in urine can be used to diagnose bladder cancer [40], and decreased levels of miR-125a and miR-200a in saliva is associated with the procession of oral squamous cell carcinoma [41]. According to the results of extracellular miRNA in blood serum and urine, they have showed that miRNA present in many other forms of body fluids including tears, saliva, seminal plasma, amniotic fluid, breast milk, colostrum, cerebrospinal fluid, bronchial lavage, peritoneal fluid, and pleural fluid [41, 42]. MiRNAs, including miR-122, miR-192, miR-21, miR-223, miR-26a, miR-27a and miR-80, provided a high diagnostic accuracy of hepatitis $B$ virus-related hepatocellular carcinoma [43]. Moreover, microRNA-501-3p could suppress metastasis and progression of hepatocellular carcinoma through targeting Lin-7 homolog A (LIN7A) [44]. Extracellular miRNA have been already detected in cell-exported exosomes. However, we found that vesicle-encapsulated miRNAs represent only a minor portion and most of extracellular miRNA interact with Ago2 protein as a RISC complex to play the role [20].
In addition, both Ago2 and Ago1-bound miRNAs can be identified in human blood plasma [45].

Besides circulating miRNAs, lncRNAs in extravesicles are novel potential biomarkers for diagnosis and prognosis. LncRNAs are a group of long noncoding RNAs with diverse functions in the cell. LncRNAs can be stable in the plasma and other body fluids and could therefore serve as biomarkers for some diseases. For example, lncRNA-ROR (linc-ROR), a stress responsive lncRNA was enriched within extracellular vesicles derived from tumor cells. It was found that increasing linc-ROR expression through vesicle reduced chemotherapy-induced cell death and modulate the intercellular response to hypoxia. These results implicate the transfer of extracellular vesicle lncRNAs take part in many intercellular responses in hepatocellular cancer cell (HCC) as mediators $[3,46]$. Another lncRNA, HULC highly expressed in the HCC patients' plasma and associated with higher Edmondson histological grades [47]. The ucRNA (ultranconserved lncRNA) TUC399 was identified to express in exosomes of HCC lines, and exosomal TUC399 that transferred into the cell can contribute to tumor growth and progression [48]. Moreover, The LINC01138 drives malignancies via activating arginine methyltransferase 5 in HCC [49], while long non-coding RNA00607 could act as a tumor suppressor by modulating NF- $\mathrm{kB}$ p65/ p53 signaling axis in HCC [50]. The lncRNA PCA3 can be identified in urine samples as a specific disease marker in prostate cancer $[51,52]$.

It was also shown that miR-210 in EVs can induce the activation of endothelial cell [53]. Moreover, miR-210 expression was inversely correlated with a disease-free and overall survival in breast cancer [54]. Epstein-Barr virus (EBV) encodes miRNAs, as the first reported viral miRNAs in human, play important roles in inhibiting apoptosis and suppressing the host immune response [55]. Some studies have reported that miRNAs in apoptotic bodies were transferred between the cells. For example, miR-126, which was contained in endothelial cell-derived apoptotic bodies, promoted endothelial cell proliferation and contributed in the angiogenesis properties [56]. In addition, it is reported that EPC-derived EVs can induce neoangiogenesis and enhance recovery in a hind limb ischemia [57].

\section{Extracellular RNA and SLE}

Systemic lupus erythematosus (SLE) is a severe autoimmune disease with various clinical manifestations including multiorgan involvement, an unpredictable course with alternation of flares and remissions requiring long-term treatment with glucocorticoids, immunosuppressive and biologic agents or both. The worldwide overall incidence rates of SLE range from 1 to 10 per 100,000 person-years, and SLE affects predominantly 
females (the female to male ratio is 9:1) in reproductive age [58]. The aetiology of SLE is multifactorial, including but not limiting contributions from the environment, stochastic factor, and genetic susceptibility.

\section{Extracellular RNA in SLE pathogenesis miRNA and SLE pathogenesis}

For the deep understanding the function of miRNAs and their target proteins, researchers found that many miRNAs can affect the immune system by directly working on innate and adaptive immune cells. Monocytes from active lupus patients expressed significant lower miRNA-302d, which is associated with increased interferon regulatory factor (IRF)-9 both in patients and pristine induce lupus model [59]. Moreover, in kidney tissues of lupus patients and mice model, renal mesangial cells express much lower miRNA-302d, which is negatively correlated with its target gene IRF-1 [60]. Cheng, et al. found that miRNA-451a increased in both spleen and thymus tissues in lupus mice model. The deficiency of miRNA-451a decreased proteinuria and immune complex deposit, concomitant with the decrease of $\mathrm{CD}^{+} \mathrm{CD}^{+} 9^{+}$and $\mathrm{CD} 4 / \mathrm{CD} 8 \mathrm{~T}$ cell ratio. This effect was mediated by inhibiting miRNA-451a target gene, IRF-8 [61]. In active SLE patient peripheral CD4 ${ }^{+} \mathrm{T}$ cells, both miR-148a and miR-126 were increased and directly target DNA methyltransferase 1 (DNMT1), then cause DNA hypomethylation and increasing CD11a and CD70 expressions on T cells. This effect can promote the autoreactivity of $\mathrm{CD}^{+}{ }^{+} \mathrm{T}$ cells in SLE patients $[62,63] . \mathrm{CD}^{+}$ $\mathrm{T}$ cells from active lupus patients showed an increase of miR-873. The further mechanism study confirmed that miR-873 facilitated the Th17 cell differentiation from naïve $\mathrm{CD}^{+} \mathrm{T}$ cells by targeting forkhead box $\mathrm{O} 1$ (Foxo1), which is known as an inhibitor of Th17 cell differentiation. In MRL/lpr lupus prone mice model, inhibition of miR-873 treatment resulted in decreased proteinuria and serum autoantibodies, as well as IL-17 expressions [64], suggesting the potential role of miR-873 as a target for lupus treatment.

For B cell targeted miRNAs studies, miR-1246 expression was significantly decreased in B cells from active SLE compared to healthy controls. Transfection of miR-1246 inhibitors into B cells markedly upregulated the expression of EBF1, and enhanced B cell function, and increased the CD40, CD80, and CD86 production on the surface of B cells [65]. In another study the authors found that miR-30a level is upregulated in CD19 ${ }^{+}$ B cells from patients with SLE, and was negatively correlated with Lyn expression, which is a target gene of miR-30a. Moreover, Lyn is also a negative regulator of $B$ cell activation. Other studies also found that miR-15a, miR-155 and miR-181b are involved in B cell activation in SLE $[65,66]$. In active SLE patients, miRNA-326 significantly promoted peripheral $\mathrm{CD} 19^{+} \mathrm{B}$ cells to differentiate into $\mathrm{CD}_{138^{+}}$plasma cells as well as anti-dsDNA expression. They further showed that miRNA-326 promoted B cells hyperactivity via Ets-1, a negative regulator of $B$ cell differentiation [67]. In pediatric lupus patients, PBMC PU.1, a target for miRNA-155, significantly upregulated and directly correlated with disease activity. Ectopic expression of miR-155 and knockdown of PU.1 suppressed TNF- $\alpha$ and BAFF on $\mathrm{CD} 19^{+} \mathrm{B}$ cells. This study provided an evidence of miRNA-155 suppression of autoimmunity through transcriptional repression of PU.1, TNF- $\alpha$ and BAFF on lupus B cells [68].

MiRNAs are not only located intracelullarly, but their presence in tissues and body fluids, such as the plasma and the serum $[69,70]$, makes them potential blood-based biomarkers of disease development and activity as well as for predicting therapeutic responses. In mice spleen cells, apoptotic lymphocytes are most likely to be the contributor of extracellular RNA. The ratio of RNA in spleen supernatant to total RNA in BALB/c mice was negatively correlated with age (from 3 weeks of approximately 70 to $45 \%$ at 6 months). However, in a BXSB lupus animal model, this ratio remained at about $70 \%$ irrespective of age. RNase activity in the spleen supernatant of 6-month-old BXSB mice was significantly lower than that of $\mathrm{BALB} / \mathrm{c}$ mice. This is the early evidence to show the correlation between extracellular RNA and autoimmune disease, especially lupus model [71]. In SLE, different patterns of miRNAs expression have been detected in the plasma, serum and urine, as well as in peripheral mononuclear cells (PBMC). As an important player in immune regulation, miRNA dysregulation is substantially involved in SLE pathogenesis. Interestingly, under expression of several miRNAs is more common in SLE [72-74]. Recently Zhang et al. screened SLE B cell related miRNAs in plasma by using miRNA PCR Array and found 14 miRNAs differently expressed between SLE patients and healthy donors and acted as the diagnostic signature. They also found $6 \mathrm{miR}$ NAs were significantly and differently expressed between SLE and RA patients. Moreover, they found that the downregulation of miR-15b, miR-93, miR-25, and miR-9b was associated with disease activity of SLE, providing evidence that circulating miRNA can be used as biomarker for SLE diagnosis [75]. EVs predominantly express many extracellular miRNAs, and miR-21 that encapsulated by EVs can act as an endogenous ligand and induction of both TLR8 and cytokine expression. This study elucidated a novel innate inflammatory pathway in SLE [76].

\section{LncRNA and SLE pathogenesis}

Many studies have shown that lncRNA and their potential correlation with autoimmune disease activity and 
progression. For the observation of lncRNAs on different immune cells in SLE, Wang et al. recruited 15 female lupus patients and 15 healthy controls. By comparing peripheral monocytes derived dendritic cells (moDCs), they found 118 lncRNAs upregulated and 45 downregulated according to lncRNA microarray analysis in moDCs of SLE patients. The expression levels of LINC00013 and NEAT1 were positively correlated with disease activity score, respectively. This study indicated a different expression of lncRNAs in moDCs, which involved in SLE pathogenesis [77]. And, IncRNA MALAT-1, which significantly upregulated in SLE monocytes, is a pivotal regulator in SLE development and provided novel target for therapeutic intervention [78]. Human monocytes IncRNA NEAT1 was highly expressed in lupus patients and positively correlated with disease activity. NEAT1 was involvement in TLR4-mediated inflammatory process, which was through the late MAPK signaling pathway activation. These findings suggested monocytes lncRNA NEAT1 as a novel regulator in SLE patients [79]. In lupus patient PBMC lncRNA array analysis, 8868 lncRNAs were found differentially expressed between patients and controls. Through GO analysis, the downregulated and upregulated transcripts of lncRNAs were identified to be associated with biological processes, cellular components and molecular function. For detecting lncRNA profile of peripheral CD4 ${ }^{+} \mathrm{T}$ cells in SLE patient, $\mathrm{Li}$, et al. found 1935 lncRNAs and 1977 mRNAs were differentially expressed between SLE patients and healthy controls. Further results demonstrated that the expression of both uc001ykl.1 and ENST00000448942 were significantly correlated with erythrocyte sedimentation rate (ESR) [80]. Besides adult SLE, Li, et al. reported that in children SLE patients, 1042 lncRNA were found differentially expressed compared to control children.

Recently, Wu, et al. compared plasma 5 lncRNA (HOTAIRM1, lnc-DC, linc0949, GAS5 and linc0597) levels between lupus patients and healthy control. They found that plasma linc0597 was upregulated, but GAS5 and lnc-DC were decreased in human lupus. Then they divided lupus patients into two groups, with lupus nephritis (LN) and without LN. Lnc-DC was significantly higher in LN patients. Further analysis demonstrated that linc0597, GAS5 and lnc-DC may specifically identify lupus. Moreover, linc0597 and GAS5 together provided better diagnostic significance. This study suggested plasma lnc-DC linc0597 and GAS5 could be potential biomarkers for lupus patients [81].

\section{Extracellular RNA and organ involvement in SLE}

Renal involvement is the most common and LN is the main cause of mortality for human SLE [82]. The expression of serum miRNA-151a-3p was significantly down-regulated in the class IV LN group compared to that in the control group and was negatively correlated with disease activity index, suggesting miRNA-151a-3p can be employed for diagnosing class IV LN [83]. However, this study did not involve and compare other types of LN patients. Su, et al. then adopted NGS for miRNAs between patients with or without LN, and finally found that miR-146a-5p had a significant correlation with clinical biochemistry markers, which can be used as a surrogate biomarker for the early detection of LN [84]. MiR-130b was downregulated in hormone-resistant LN patients and it inhibited the viability but accelerated the apoptosis of mesangial cells via its target gene PTEN, phosphatase and tensin homolog [85]. MiR-22 and miR-15b expressions were significantly lower in lupus with low estimate glomerular filtration rate (eGFR) [75]. One study involved 16 SLE patients, including 9 with LN and 7 without. They found that serum has-miR-5571-5p and has-miR-766-3p significantly decreased in LN patients compared those without LN [86].

For urine miRNA profiles between LN and diabetic nephropathy patients, Cardenas et al. found that miR-1273e and miR-3201 were downregulated and associated with endocapillary glomerular inflammation. However, in diabetic nephropathy, miR-4532, miR-1915-3p and miR-2861 were downregulated and associated with estimated glomerular filtration rate and interstitial fibrosis/tubular atrophy. This study for the first time showed that miRNAs can be used as biomarkers to distinguish LN and diabetic nephropathy [87].

The high risk of both subclinical atherosclerosis and cardiovascular events are well established in SLE. Antiphospholipid syndrome (APS) secondary to SLE also showed an increased risk of developing cardiovascular diseases in patients [88]. For the observation of different miRNAs profiles in a subset of lupus patients who had cardiovascular events, Kay et al. identified that miR-101, miR-375 and miR-125b were indicative of atherosclerosis, with a sensitivity of $86 \%$ and a specificity of $88 \%$. They also found an eight-miRNA profile that differentiated a subgroup of SLE with venous thrombotic events, thrombocytopenia and $\beta 2$-glycoprotein I antibodies [89]. Recently, the miRNA profile was assessed in SLE pDCs, APS secondary to SLE (SLE + APS) and primary APS (PAPS) patients, but the result showed that the whole miRNA profiles of SLE, SLE + APS and PAPS did not show any difference, as well as IFN score among the three groups [90], so the detailed role of miRNA in SLE cardiovascular events needs further investigation.

Pleuritis and pericarditis are observed in late-onset lupus patients. The TRAF3IP2 gene polymorphisms have been observed in association with SLE susceptibility and pericarditis development. TRAF3IP2 is the target gene of miR1279. Ciccacci's group used allelic discrimination assay for Italian lupus patients and found miR1279 was 
a useful predictor for pericarditis development in SLE patients [91]. Huang et al. found that miR-210 expression significantly increased in lupus patients and especially in those with pleuritis and positive anti-SSB/La antibody [92].

\section{Extracellular RNA and SLE treatment}

For the important role of extracellular RNA in lupus pathogenesis, the expression declined after effective therapy. SLE patient plasma miR-6088, miR-4732-5p and miR-1246 were significantly decreased after passage through the plasma adsorption membrane [93]. Mesenchymal stem cells (MSC) showed encouraging potential for lupus treatment. Lupus patient bone marrow derived MSC expressed significant higher level of miR-663, which mediated the inhibition of MSC proliferation, migration and enhancement of its apoptosis, as well as the immunoregulatory properties. MiR-663 is targeting transforming growth factor $\beta 1$ (TGF- $\beta 1$ ). MiR-663 overexpression weakened MSC therapeutic effect, while miR-663 inhibition improves disease remission in lupus mice. So miR-663 is an important mediator of SLE bone marrow MSC regulation and

Table 1 The change of key extracellular RNAs in SLE patients

\begin{tabular}{|c|c|c|}
\hline exRNA (human) & exRNA expression change & exRNA in disease \\
\hline miR-451a & $\bar{\uparrow}$ & SLE pathogenensis \\
\hline miR-148a & $\uparrow$ & \\
\hline miR-126 & $\uparrow$ & \\
\hline miR-873 & $\uparrow$ & \\
\hline miR-1246 & $\downarrow$ & \\
\hline miR-30a & $\uparrow$ & \\
\hline miR-326 & $\uparrow$ & \\
\hline miR-15b & $\downarrow$ & \\
\hline $\operatorname{miR}-25$ & $\downarrow$ & \\
\hline miR-302 & $\downarrow$ & \\
\hline miR-93 & $\downarrow$ & \\
\hline miR-9b & $\downarrow$ & \\
\hline Linc00013 & $\uparrow$ & \\
\hline NEAT-1 & $\uparrow$ & \\
\hline MALAT-1 & $\uparrow$ & \\
\hline GAS5 & $\downarrow$ & \\
\hline Linc0597 & $\uparrow$ & \\
\hline Inc-DC & $\downarrow$ & \\
\hline miR-22 & $\downarrow$ & Lupus nephritis \\
\hline miR-15b & $\downarrow$ & \\
\hline miR-151a-3p & $\downarrow$ & \\
\hline miR-130b & $\downarrow$ & \\
\hline miR-5571-5p & $\downarrow$ & \\
\hline miR-766-3p & $\downarrow$ & \\
\hline
\end{tabular}

may serve as a new therapeutic target for lupus treatment [94]. Human adipose tissue derived MSC showed obvious amelioration of lupus mice model, by reducing serum anti-dsDNA levels, glomerular C3 deposition and trabecular damage. The splenocyte miR-182-5p and miR-96-5p significantly decreased, accompanied by decreased CD138 proportion and Th1/Th2 ratio after MSC treatment [95].

\section{Conclusions}

Though the long-term prognosis and survival rate of lupus has significantly improved, significant morbidity and mortality are still associated to a substantial extent. And, disease pathogenesis is still not clear. Since the recent discovery, the noncoding RNA such as miRNA and IncRNA have been reported to play an important role in autoimmune disorders. MiRNAs and lncRNAs are not located only intracelullarly, but their presence in tissues and body fluids, such as the plasma and the serum, makes them potential blood-based biomarkers of disease development and activity as well as for predicting therapeutic responses. In this review, we summarize the current state and function of exRNAs in SLE (Table 1). exRNAs may take parts in the process of SLE and some of them can be used as diagnostic tool for SLE.

\section{Abbreviations}

APS: Antiphospholipid syndrome; circRNA: circular RNAs; CRP: C reactive protein; CSF: Cerebrospinal fluid; DNMT1: DNA methyltransferase 1; EBF1: Early B cell factor 1; EBV: Epstein-Barr virus; ESR: Erythrocyte sedimentation rate; EVs: Extracellular vesicles; exRNA: extracellular RNA; HCC: Hepatocellular cancer cell; IRF: Interferon regulatory factor; lincROR: IncRNA-ROR; LN: Lupus nephritis; IncRNA: long non-coding RNAs; LPPs: Lipoproteins; miRNA: microRNAs; moDCs: monocytes derived dendritic cells; MSC: Mesenchymal stem cells; ncRNA: non-coding RNAs; NGS: Nextgeneration sequencing; PAPS: Primary antiphospholipid syndrome; PBMC: Peripheral mononuclear cells; piRNA: piwi-RNAs;

RNPs: Ribonucleoprotein particles; rRNA: ribosomal RNAs; SLE: Systemic lupus erythematosus; snoRNA: small nucleolar RNAs; snRNA: small nuclear RNAs;

TGF- $\beta 1$ : Transforming growth factor $\beta 1$; tRNA: transfer RNAs;

ucRNA: Ultranconserved IncRNA

\section{Acknowledgements}

Not applicable.

\section{Funding}

This paper was funded by the National Natural Science Foundation of China (31700719), the Jiangsu Province Six Talent Project (2016-WSN-156), and the Nanjing Outstanding Youth Foundation (JQX17004).

Availability of data and materials

Pubmed.

Authors' contributions

Both SL and DW contribute to the writing and approval of publication of this review. All authors read and approved the final manuscript.

Ethics approval and consent to participate

Not applicable.

Consent for publication

Both authors approved to publish this review. 


\section{Competing interests}

The authors declare that they have no competing interest.

\section{Publisher's Note}

Springer Nature remains neutral with regard to jurisdictional claims in published maps and institutional affiliations.

\section{Author details}

'Department of Rheumatology and Immunology, the Affiliated Drum Tower Hospital of Nanjing University Medical School, Nanjing 210008, China. ${ }^{2}$ Program of Immunology \& Molecular Cellular Biology, Department of Biology, Center for Diagnostics \& Therapeutics, Center of Inflammation, Immunity \& Infection, Georgia State University, Atlanta, GA 30302, USA.

\section{Received: 3 October 2018 Accepted: 15 March 2019}

\section{Published online: 08 November 2019}

\section{References}

1. Huang X, Yuan T, Tschannen M, et al. Characterization of human plasmaderived exosomal RNAs by deep sequencing. BMC Genomics. 2013;14:319.

2. Li Y, Zheng Q, Bao C, et al. Circular RNA is enriched and stable in exosomes: a promising biomarker for cancer diagnosis. Cell Res. 2015;25(8):981-4.

3. Takahashi K, Yan IK, Kogure T, et al. Extracellular vesicle-mediated transfer of long non-coding RNA ROR modulates chemosensitivity in human hepatocellular cancer. FEBS Open Bio. 2014;4:458-67.

4. Skog J, Wurdinger T, van Rijn S, et al. Glioblastoma microvesicles transport RNA and proteins that promote tumour growth and provide diagnostic biomarkers. Nat Cell Biol. 2008;10(12):1470-6.

5. Gezer U, Ozgur E, Cetinkaya M, et al. Long non-coding RNAs with low expression levels in cells are enriched in secreted exosomes. Cell Biol Int. 2014;38(9):1076-9.

6. Yuan $T$, Huang $X$, Woodcock $M$, et al. Plasma extracellular RNA profiles in healthy and cancer patients. Sci Rep. 2016;6:19413.

7. Bahn JH, Zhang Q, Li F, et al. The landscape of microRNA, Piwi-interacting RNA, and circular RNA in human saliva. Clin Chem. 2015;61(1):221-30.

8. Ben-Dov IZ, Whalen VM, Goilav B, et al. Cell and microvesicle urine microRNA deep sequencing profiles from healthy individuals: observations with potential impact on biomarker studies. PLoS One. 2016;11(1):e0147249.

9. Dhahbi JM, Spindler SR, Atamna H, et al. 5' tRNA halves are present as abundant complexes in serum, concentrated in blood cells, and modulated by aging and calorie restriction. BMC Genomics. 2013;14:298.

10. Vojtech L, Woo S, Hughes S, et al. Exosomes in human semen carry a distinctive repertoire of small non-coding RNAs with potential regulatory functions. Nucleic Acids Res. 2014;42(11):7290-304

11. Wang K, Li H, Yuan Y, et al. The complex exogenous RNA spectra in human plasma: an interface with human gut biota? PLoS One. 2012;7(12):e51009.

12. Leung RK, Wu YK. Circulating microbial RNA and health. Sci Rep. 2015:5: 16814.

13. Dhahbi JM, Spindler SR, Atamna $\mathrm{H}$, et al. Deep sequencing of serum small RNAs identifies patterns of $5^{\prime}$ tRNA half and YRNA fragment expression associated with breast Cancer. Biomark Cancer. 2014;6:37-47.

14. Sato-Kuwabara Y, Melo SA, Soares FA, et al. The fusion of two worlds: noncoding RNAs and extracellular vesicles--diagnostic and therapeutic implications (review). Int J Oncol. 2015:46(1):17-27.

15. Chen $X$, Liang $H$, Zhang J, et al. Secreted microRNAs: a new form of intercellular communication. Trends Cell Biol. 2012:22(3):125-32.

16. Burgos $\mathrm{KL}$, Javaherian A, Bomprezzi $R$, et al. Identification of extracellular miRNA in human cerebrospinal fluid by next-generation sequencing. Rna. 2013;19(5):712-22.

17. Vickers KC, Palmisano BT, Shoucri BM, et al. MicroRNAs are transported in plasma and delivered to recipient cells by high-density lipoproteins. Nat Cell Biol. 2011;13(4):423-33.

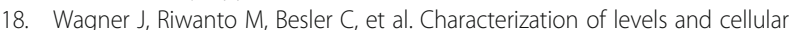
transfer of circulating lipoprotein-bound microRNAs. Arterioscler Thromb Vasc Biol. 2013:33(6):1392-400.

19. Arroyo JD, Chevillet JR, Kroh EM, et al. Argonaute2 complexes carry a population of circulating microRNAs independent of vesicles in human plasma. Proc Natl Acad Sci U S A. 2011;108(12):5003-8.

20. Turchinovich $A$, Weiz $L$, Langheinz $A$, et al. Characterization of extracellular circulating microRNA. Nucleic Acids Res. 2011;39(16):7223-33.
21. Holme PA, Solum NO, Brosstad F, et al. Demonstration of platelet-derived microvesicles in blood from patients with activated coagulation and fibrinolysis using a filtration technique and western blotting. Thromb Haemost. 1994;72(5):666-71.

22. Hess JR. Blood substitutes for surgery and trauma: efficacy and toxicity issues. BioDrugs. 1999;12(2):81-90.

23. Cocucci E, Racchetti G, Meldolesi J. Shedding microvesicles: artefacts no more. Trends Cell Biol. 2009;19(2):43-51.

24. Gyorgy K, Ajtony Z, Van Meel K, et al. Fast heating induced impulse halogenation of refractory sample components in electrothermal atomic absorption spectrometry by direct injection of a liquid halogenating agent. Talanta. 2011;85(3):1253-9.

25. Loyer $\mathrm{X}$, Vion AC, Tedgui A, et al. Microvesicles as cell-cell messengers in cardiovascular diseases. Circ Res. 2014;114(2):345-53.

26. Colombo M, Raposo G, Thery C. Biogenesis, secretion, and intercellular interactions of exosomes and other extracellular vesicles. Annu Rev Cell Dev Biol. 2014:30:255-89.

27. Zernecke A, Bidzhekov K, Noels H, et al. Delivery of microRNA-126 by apoptotic bodies induces CXCL12-dependent vascular protection. Sci Signal. 2009;2(100):ra81.

28. Tkach $\mathrm{M}$, Thery $\mathrm{C}$. Communication by extracellular vesicles: where we are and where we need to go. Cell. 2016;164(6):1226-32.

29. Raposo G, Stoorvogel W. Extracellular vesicles: exosomes, microvesicles, and friends. J Cell Biol. 2013;200(4):373-83.

30. Song J, Chen X, Wang M, et al. Cardiac endothelial cell-derived exosomes induce specific regulatory B cells. Sci Rep. 2014;4:7583.

31. Hergenreider E, Heydt S, Treguer K, et al. Atheroprotective communication between endothelial cells and smooth muscle cells through miRNAs. Nat Cell Biol. 2012;14(3):249-56.

32. Valadi $\mathrm{H}$, Ekstrom $\mathrm{K}$, Bossios $\mathrm{A}$, et al. Exosome-mediated transfer of mRNAs and microRNAs is a novel mechanism of genetic exchange between cells. Nat Cell Biol. 2007:9(6):654-9.

33. Fiandaca MS, Kapogiannis D, Mapstone M, et al. Identification of preclinical Alzheimer's disease by a profile of pathogenic proteins in neurally derived blood exosomes: a case-control study. Alzheimers Dement. 2015;11(6):600-7 e601.

34. Smith JA, Leonardi $T$, Huang $B$, et al. Extracellular vesicles and their synthetic analogues in aging and age-associated brain diseases. Biogerontology. 2015;16(2):147-85.

35. Gaceb A, Martinez MC, Andriantsitohaina R. Extracellular vesicles: new players in cardiovascular diseases. Int J Biochem Cell Biol. 2014:50:24-8.

36. Zhang L, Zhang S, Yao J, et al. Microenvironment-induced PTEN loss by exosomal microRNA primes brain metastasis outgrowth. Nature. 2015; 527(7576):100-4.

37. Catto JW, Miah S, Owen HC, et al. Distinct microRNA alterations characterize high- and low-grade bladder cancer. Cancer Res. 2009;69(21):8472-81.

38. Lodes MJ, Caraballo M, Suciu D, et al. Detection of cancer with serum miRNAs on an oligonucleotide microarray. PLoS One. 2009:4(7):e6229.

39. Mitchell PS, Parkin RK, Kroh EM, et al. Circulating microRNAs as stable blood-based markers for cancer detection. Proc Natl Acad Sci U S A. 2008;105(30):10513-8.

40. Hanke M, Hoefig K, Merz $\mathrm{H}$, et al. A robust methodology to study urine microRNA as tumor marker: microRNA-126 and microRNA-182 are related to urinary bladder cancer. Urol Oncol. 2010;28(6):655-61.

41. Park NJ, Zhou H, Elashoff D, et al. Salivary microRNA: discovery, characterization, and clinical utility for oral cancer detection. Clin Cancer Res. 2009;15(17):5473-7.

42. Weber JA, Baxter DH, Zhang S, et al. The microRNA spectrum in 12 body fluids. Clin Chem. 2010:56(11):1733-41.

43. Zhou J, Yu L, Gao X, et al. Plasma microRNA panel to diagnose hepatitis B virus-related hepatocellular carcinoma. J Clin Oncol. 2011;29(36):4781-8.

44. Luo $C$, Yin D, Zhan H, et al. microRNA-501-3p suppresses metastasis and progression of hepatocellular carcinoma through targeting LIN7A. Cell Death Dis. 2018:9(5):535.

45. Turchinovich A, Weiz L, Burwinkel B. Extracellular miRNAs: the mystery of their origin and function. Trends Biochem Sci. 2012;37(11):460-5.

46. Takahashi $\mathrm{K}$, Yan IK, Haga H, et al. Modulation of hypoxia-signaling pathways by extracellular linc-RoR. J Cell Sci. 2014;127(Pt 7):1585-94.

47. Xie H, Ma H, Zhou D. Plasma HULC as a promising novel biomarker for the detection of hepatocellular carcinoma. Biomed Res Int. 2013:2013:136106.

48. Kogure $T$, Yan IK, Lin WL, et al. Extracellular vesicle-mediated transfer of a novel Long noncoding RNA TUC339: a mechanism of intercellular signaling in human hepatocellular Cancer. Genes Cancer. 2013;4(7-8):261-72. 
49. Li Z, Zhang J, Liu X, et al. The LINC01138 drives malignancies via activating arginine methyltransferase 5 in hepatocellular carcinoma. Nat Commun. 2018;9(1):1572

50. Sun QM, Hu B, Fu PY, et al. Long non-coding RNA00607 as a tumor suppressor by modulating NF-kB p65/p53 signaling axis in hepatocellular carcinoma. Carcinogenesis. 2018. https://doi.org/10.1093/carcin/bgy113 [Epub ahead of print].

51. Panzitt K, Tschernatsch MM, Guelly C, et al. Characterization of HULC, a novel gene with striking up-regulation in hepatocellular carcinoma, as noncoding RNA. Gastroenterology. 2007;132(1):330-42.

52. Yang Y, Cai Y, Wu G, et al. Plasma long non-coding RNA, CoroMarker, a novel biomarker for diagnosis of coronary artery disease. Clin Sci. 2015;129(8):675-85.

53. Kosaka N, Iguchi $H$, Hagiwara $K$, et al. Neutral sphingomyelinase 2 (nSMase2)-dependent exosomal transfer of angiogenic microRNAs regulate cancer cell metastasis. J Biol Chem. 2013;288(15):10849-59.

54. Camps C, Buffa FM, Colella S, et al. Hsa-miR-210 is induced by hypoxia and is an independent prognostic factor in breast cancer. Clin Cancer Res. 2008; 14(5):1340-8.

55. Forte $\mathrm{E}$, Luftig MA. The role of microRNAs in Epstein-Barr virus latency and lytic reactivation. Microbes Infect. 2011;13(14-15):1156-67.

56. Cantaluppi V, Biancone L, Figliolini F, et al. Microvesicles derived from endothelial progenitor cells enhance neoangiogenesis of human pancreatic islets. Cell Transplant. 2012;21(6):1305-20.

57. Ranghino A, Cantaluppi V, Grange C, et al. Endothelial progenitor cellderived microvesicles improve neovascularization in a murine model of hindlimb ischemia. Int J Immunopathol Pharmacol. 2012;25(1):75-85.

58. Frieri M. Mechanisms of disease for the clinician: systemic lupus erythematosus. Ann Allergy Asthma Immunol. 2013;110(4):228-32.

59. Smith S, Fernando T, Wu PW, et al. MicroRNA-302d targets IRF9 to regulate the IFN-induced gene expression in SLE. J Autoimmun. 2017;79:105-11.

60. Han $X$, Wang $Y$, Zhang $X$, et al. MicroRNA-130b ameliorates murine lupus nephritis through targeting the type I interferon pathway on renal mesangial cells. Arthritis Rheumatol. 2016;68(9):2232-43.

61. Cheng J, Wu R, Long $L$, et al. miRNA-451a targets IFN regulatory factor 8 for the progression of systemic lupus erythematosus. Inflammation. 2017;40(2):676-87.

62. Pan W, Zhu S, Yuan M, et al. MicroRNA-21 and microRNA-148a contribute to DNA hypomethylation in lupus CD4+ T cells by directly and indirectly targeting DNA methyltransferase 1. J Immunol. 2010;184(12):6773-81.

63. Zhao $S$, Wang $Y$, Liang $Y$, et al. MicroRNA-126 regulates DNA methylation in CD4+ T cells and contributes to systemic lupus erythematosus by targeting DNA methyltransferase 1. Arthritis Rheum. 2011;63(5):1376-86.

64. Liu L, Liu Y, Yuan M, et al. Elevated expression of microRNA-873 facilitates Th17 differentiation by targeting forkhead box $\mathrm{O} 1$ (Foxo1) in the pathogenesis of systemic lupus erythematosus. Biochem Biophys Res Commun. 2017;492(3):453-60

65. Luo S, Liu Y, Liang G, et al. The role of microRNA-1246 in the regulation of $B$ cell activation and the pathogenesis of systemic lupus erythematosus. Clin Epigenetics. 2015;7:24.

66. Long $H$, Wang $X$, Chen $Y$, et al. Dysregulation of microRNAs in autoimmune diseases: pathogenesis, biomarkers and potential therapeutic targets. Cancer Lett. 2018;428:90-103.

67. Xia $Y$, Tao $J H$, Fang $X$, et al. MicroRNA-326 upregulates B cell activity and autoantibody production in lupus disease of MRL/lpr mice. Mol Ther Nucleic Acids. 2018;11:284-91.

68. Aboelenein HR, Hamza MT, Marzouk H, et al. Reduction of CD19 autoimmunity marker on B cells of paediatric SLE patients through repressing PU.1/TNFalpha/BAFF axis pathway by miR-155. Growth Factors. 2017;35(2-3):49-60.

69. Carlsen AL, Schetter AJ, Nielsen CT, et al. Circulating microRNA expression profiles associated with systemic lupus erythematosus. Arthritis Rheum. 2013;65(5):1324-34.

70. Stagakis $E$, Bertsias $G$, Verginis $P$, et al. Identification of novel microRNA signatures linked to human lupus disease activity and pathogenesis: miR-21 regulates aberrant $T$ cell responses through regulation of PDCD4 expression. Ann Rheum Dis. 2011;70(8):1496-506.

71. Zhao WP, Liu XG, Wang W, et al. Large quantity of ribosomal RNA exists extracellularly in mouse spleen. Histochem J. 2002;34(8-9):391-6.

72. Tang $Y$, Luo $X$, Cui $H$, et al. MicroRNA-146A contributes to abnormal activation of the type I interferon pathway in human lupus by targeting the key signaling proteins. Arthritis Rheum. 2009;60(4):1065-75.

73. Dai $Y$, Huang YS, Tang M, et al. Microarray analysis of microRNA expression in peripheral blood cells of systemic lupus erythematosus patients. Lupus. 2007;16(12):939-46.
74. Husakova M. MicroRNAs in the key events of systemic lupus erythematosus pathogenesis. Biomed Pap Med Fac Univ Palacky Olomouc Czech Repub. 2016;160(3):327-42.

75. Zhang $H$, Huang $X$, Ye L, et al. B cell-related circulating MicroRNAs with the potential value of biomarkers in the differential diagnosis, and Distinguishment between the disease activity and lupus nephritis for systemic lupus erythematosus. Front Immunol. 2018;9:1473.

76. Young NA, Valiente GR, Hampton JM, et al. Estrogen-regulated STAT1 activation promotes TLR8 expression to facilitate signaling via microRNA-21 in systemic lupus erythematosus. Clin Immunol. 2017;176:12-22.

77. Wang Y, Chen S, Chen S, et al. Long noncoding RNA expression profile and association with SLEDAI score in monocyte-derived dendritic cells from patients with systematic lupus erythematosus. Arthritis Res Ther. 2018;20(1):138.

78. Yang $\mathrm{H}$, Liang $\mathrm{N}$, Wang $\mathrm{M}$, et al. Long noncoding RNA MALAT-1 is a novel inflammatory regulator in human systemic lupus erythematosus. Oncotarget. 2017:8(44):77400-6.

79. Zhang F, Wu L, Qian J, et al. Identification of the long noncoding RNA NEAT1 as a novel inflammatory regulator acting through MAPK pathway in human lupus. J Autoimmun. 2016:75:96-104.

80. Li LJ, Zhao W, Tao SS, et al. Comprehensive long non-coding RNA expression profiling reveals their potential roles in systemic lupus erythematosus. Cell Immunol. 2017;319:17-27.

81. Wu GC, Li J, Leng RX, et al. Identification of long non-coding RNAs GAS5, linc0597 and Inc-DC in plasma as novel biomarkers for systemic lupus erythematosus. Oncotarget. 2017:8(14):23650-63.

82. Zimmerman R, Radhakrishnan J, Valeri A, et al. Advances in the treatment of lupus nephritis. Annu Rev Med. 2001;52:63-78.

83. Xiao H, Wei N, Su M, et al. Down-regulation of serum miR-151a-3p is associated with renal tissue activity in class IV lupus nephritis. Clin Exp Rheumatol. 2019;37(1):67-72.

84. Su YJ, Lin IC, Wang L, et al. Next generation sequencing identifies miRNAbased biomarker panel for lupus nephritis. Oncotarget. 2018;9(46):27911-9.

85. Wu S, Wang J, Li F. Dysregulation of PTEN caused by the underexpression of microRNA130b is associated with the severity of lupus nephritis. Mol Med Rep. 2018;17(6):7966-72.

86. Jafari Ghods F, Topal Sarikaya A, Arda N, et al. MiRNA and mRNA profiling in systemic lupus reveals a novel set of cytokine - related miRNAs and their target genes in cases with and without renal involvement. Kidney Blood Press Res. 2017:42(6):1322-37.

87. Cardenas-Gonzalez M, Srivastava A, Pavkovic M, et al. Identification, confirmation, and replication of novel urinary MicroRNA biomarkers in lupus nephritis and diabetic nephropathy. Clin Chem. 2017;63(9):1515-26.

88. Giannelou M, Mavragani CP. Cardiovascular disease in systemic lupus erythematosus: a comprehensive update. J Autoimmun. 2017;82:1-12.

89. Kay SD, Carlsen AL, Voss A, et al. Associations of circulating cell-free microRNA with vasculopathy and vascular events in systemic lupus erythematosus patients. Scand J Rheumatol. 2018:1-10.

90. van den Hoogen $L L$, Rossato M, Lopes AP, et al. microRNA downregulation in plasmacytoid dendritic cells in interferon-positive systemic lupus erythematosus and antiphospholipid syndrome. Rheumatology. 2018;57(9):1669-74.

91. Ciccacci C, Perricone C, Politi C, et al. A polymorphism upstream MIR1279 gene is associated with pericarditis development in systemic lupus erythematosus and contributes to definition of a genetic risk profile for this complication. Lupus. 2017;26(8):841-8.

92. Huang Q, Chen SS, Li J, et al. miR-210 expression in PBMCs from patients with systemic lupus erythematosus and rheumatoid arthritis. Ir J Med Sci. 2018;187(1):243-9.

93. Ishibe $Y$, Kusaoi M, Murayama G, et al. Changes in the expression of circulating microRNAs in systemic lupus erythematosus patient blood plasma after passing through a plasma adsorption membrane. Ther Apher Dial. 2018;22(3):278-89.

94. Geng L, Tang X, Zhou K, et al. MicroRNA-663 induces immune dysregulation by inhibiting TGF-beta1 production in bone marrow-derived mesenchymal stem cells in patients with systemic lupus erythematosus. Cell Mol Immunol. 2019; 16(3):260-74.

95. Burge DJ, Eisenman J, Byrnes-Blake K, et al. Safety, pharmacokinetics, and pharmacodynamics of RSLV-132, an RNase-fc fusion protein in systemic lupus erythematosus: a randomized, double-blind, placebo-controlled study. Lupus. 2017;26(8):825-34. 